\title{
Standard Outpatient Re-Evaluation for Patients Not Admitted to the Hospital After Emergency Department Evaluation for Acute Abdominal Pain
}

\author{
Boudewijn R. Toorenvliet $\cdot$ Rutger F. R. Bakker • \\ Hans C. Flu · Jos W. S. Merkus · Jaap F. Hamming • \\ Paul J. Breslau
}

Published online: 5 January 2010

(C) The Author(s) 2010. This article is published with open access at Springerlink.com

\begin{abstract}
Background The aim of the present study was to investigate the efficacy and safety of standard outpatient reevaluation for patients who are not admitted to the hospital after emergency department surgical consultation for acute abdominal pain.

Methods All patients seen at the emergency department between June 2005 and July 2006 for acute abdominal pain were included in a prospective study using a structured diagnosis and management flowchart. Patients not admitted to the hospital were given appointments for re-evaluation at the outpatient clinic within $24 \mathrm{~h}$. All clinical parameters, radiological results, diagnostic considerations, and management proposals were scored prospectively.

Results Five-hundred patients were included in this analysis. For 148 patients (30\%), the final diagnosis was different from the diagnosis after initial evaluation. Eightyfive patients $(17 \%)$ had a change in management after reevaluation, and 20 of them (4\%) were admitted to the hospital for an operation. Only 6 patients $(1.2 \%)$ had a delay in diagnosis and treatment, which did not cause extra morbidity.
\end{abstract}

B. R. Toorenvliet $(\bowtie) \cdot$ H. C. Flu · J. F. Hamming

Department of Surgery, Leiden University Medical Center, P.O. Box 9600, 2300 RC Leiden, The Netherlands

e-mail: br.toorenvliet@tiscali.nl

R. F. R. Bakker - J. W. S. Merkus

Department of Surgery, HAGA Hospital, P.O. Box 40551, 2504 LN Den Haag, The Netherlands

P. J. Breslau

Medical Specialist Registration Committee, KNMG, P.O. Box

20053, 3528 BL Utrecht, The Netherlands
Conclusions Standard outpatient re-evaluation is a safe and effective means of improving diagnostic accuracy and helps to adapt management for patients that are not admitted to the hospital after surgical consultation for acute abdominal pain at the emergency department.

\section{Introduction}

Approximately $4-5 \%$ of patients evaluated at an emergency department (ED) present with acute abdominal pain [1]. Some patients that require admission for surgical or medical treatment are easily recognized. Others may present during the early stages of surgical pathology and will be difficult to distinguish from patients with mild selflimiting disease. Judgment errors in evaluating these patients with an ambivalent presentation may lead to therapeutic delay, possibly increasing morbidity and even mortality. For this reason diagnostic modalities such as ultrasound (US) and computed tomography (CT) are often used to aid in the diagnostic process and subsequent clinical decision making $[2,3]$. Cross-sectional imaging may not, however, be beneficial in the diagnosis of all patients seen in the ED for acute abdominal pain. These examinations are costly, time consuming, and, in the case of CT, subject the patient to ionizing radiation.

Another method often used for the differentiation of mild disease from more serious pathology in ambivalent cases is outpatient re-evaluation. Re-evaluation can allow the disease to present itself through natural progression, permitting surgical cases to become more typical and thus identifiable. In patients with nonspecific abdominal pain or mild nonsurgical diagnoses, the symptoms will regress, allowing the patient to be safely discharged from followup. 
The present study was performed to assess the efficacy and safety of standard outpatient re-evaluation in a large series of patients with acute abdominal pain seen in our ED but who were not considered to require a hospital admission. Our hypothesis is that serial outpatient re-evaluation for patients with equivocal abdominal pain is safe, can improve diagnostic accuracy, and will facilitate proper treatment selection.

\section{Patients and methods}

The present study was performed in a middle-sized teaching hospital with a $24 \mathrm{~h}$ emergency service with surgery, radiology, intensive care, and on call consultants in pediatrics, gynecology, and internal medicine. All consecutive patients with acute abdominal pain evaluated in the ED by a resident of the surgical department between June 2005 and July 2006 were included in the study. The surgical resident always made the primary assessment, and the consultant surgeon evaluated the patient if necessary. Patients who were evaluated at another hospital for the same complaint, patients with abdominal pain caused by trauma, and patients who had undergone radiological examination (US or CT) prior to surgical consultation were excluded. For all patients, a structured diagnostic and management strategy algorithm was followed (Fig. 1). First, a "clinical diagnosis" (D1) was made based on the patient's history, physical examination, and biochemical blood and urine analyses. The resident recorded his/her degree of certainty for the clinical diagnosis given on a scale from 1 to 5 . An initial management proposal (S1) was then made based on the clinical diagnosis. All clinical parameters, the clinical diagnosis (D1), and the proposed strategy (S1) were registered on a study form. After a conference with the consulting surgeon, a decision was made about whether or not to perform additional radiological examinations. When such studies were performed, the radiologist was asked to confirm the clinical diagnosis or provide an alternative diagnosis. All US and CT examinations were performed by 1 of 5 certified radiologists with similar levels of experience. After learning the radiological results (RD1), the resident and the surgeon reassessed the initial clinical diagnosis and strategy, which were altered if necessary (CD1 \& CS1). Again all results and considerations were registered on the study form. Patients were admitted to the surgical ward if they were thought to have an abdominal condition that required immediate operation or a medical therapy necessitating admission. All patients that were not directly admitted to the surgical ward after surgical consultation at the ED were given appointments for re-evaluation at the outpatient clinic within $24 \mathrm{~h}$. There, the diagnosis and management strategies were reassessed (D2 and S2) by the consultant surgeon or a surgical resident under the supervision of a consultant surgeon. Additional radiological or endoscopic examinations were made if they were deemed necessary. Patients were discharged from out-patient follow-up when a definitive diagnosis was made and the treatment was successfully initiated or completed, or if the patient no longer had abdominal complaints. The final diagnosis (FD) was based on intraoperative findings or pathological
Fig. 1 Study design for patients presenting with abdominal pain at the emergency department for surgical consultation.

(Reprinted with permission Wiley-Blackwell Publishing.)

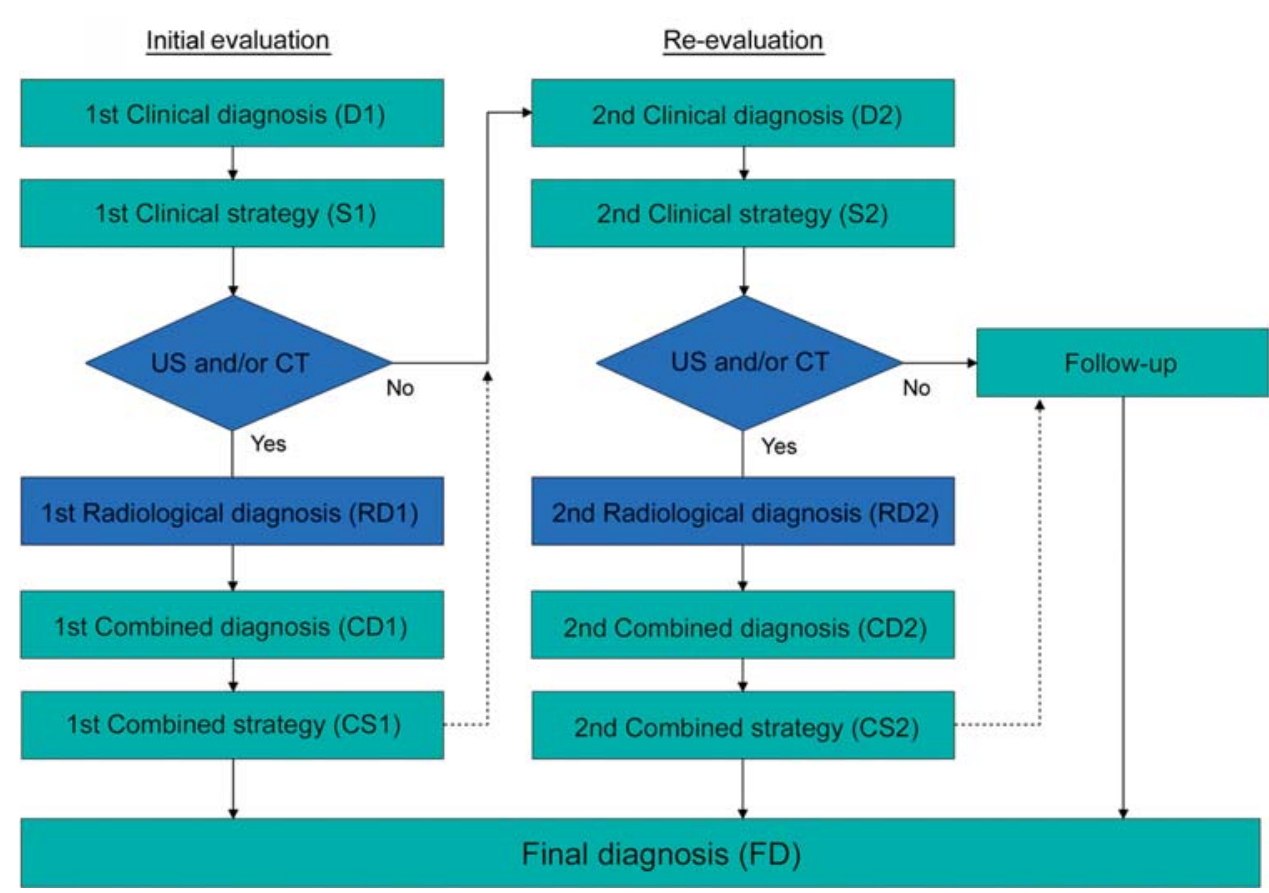


examination of the resected organs. If patients were not operated, the final diagnosis was made from the clinical and/or radiological diagnosis in combination with the clinical response to medical therapy at standard re-evaluation and follow-up as described above.

For the purposes of the present study, all hospital records were reviewed by two surgical residents (B. T. and R. B.), double-checking the available information and verifying the final diagnoses for all patients entered into the database. Patients were excluded from analysis if they did not show up for the re-evaluation appointment or if the study form was not returned or was incomplete. For all these patients, the hospital records were searched and patients were contacted for additional information. If a patients could not be contacted, that patient's general practitioner was consulted. All diagnoses were categorized according to the 10th version of the International Classification of Diseases (10-ICD) [4]. Complications noted during the hospital admission were scored twice daily in a prospective database as reported earlier [5]. Statistical analysis was performed with SPSS 16.0. Chi square tests were used to compare binomial proportions with the Yates continuity correction; $P<0.05$ was considered significant.

\section{Results}

During the study period 972 patients were evaluated. Fortynine patients $(5.0 \%)$ were excluded when they did not show up for their re-evaluation appointment, and another 121 (12.4\%) patients were excluded as the study forms were incomplete or not returned. Twenty-three patients were lost to follow-up (2.4\%). The diagnosis and management data for excluded patients are shown in Table 1 . Of the 802 patients eligible for inclusion, 302 patients (37.7\%) were admitted to the hospital, and $123(15.3 \%)$ underwent operation on the day of first evaluation. The other 500 patients (62.3\%) were not admitted and were re-evaluated according to the study protocol (Fig. 2).

All patients were evaluated by one of 16 surgical residents with different levels of experience. Eighty-eight percent of the patients, however, were seen by residents in their first 3 years of training.

Of the 500 patients enrolled in the study, 205 patients were under 17 years old $(41.0 \%)$ and 290 patients were female $(58.0 \%)$. Thirty-nine patients $(7.8 \%)$ had a fever defined as a temperature above $38^{\circ} \mathrm{C}, 160$ patients $(32.0 \%)$ had a C-reactive protein (CRP) level higher than 8, and 111 patients $(22.2 \%)$ had a leukocyte count of more than 12 . Plain abdominal radiographs were acquired in 211 patients (42.2\%), and 31 (6.2\%) had chest x-rays. A US study of the abdomen was done in 139 patients $(27.8 \%)$, a CT of the abdomen was done in $8(1.6 \%)$, and 17 patients $(3.4 \%)$ underwent both an US and a CT at initial evaluation. At reevaluation 145 patients (29.0\%) had an US, 15 (3.0\%) had a CT, and $14(2.8 \%)$ had both an US and a CT. Sixteen patients (3.2\%) underwent additional cross-sectional imaging at the initial evaluation as well as at re-evaluation.

The final diagnoses for the 500 patients that had a standard re-evaluation are given in Table 2. The average follow-up was 12 days (range: 1-275 days), but most of the patients $(46.2 \%)$ could be discharged from follow-up after just one outpatient visit.

For 148 patients $(29.6 \%)$ the final diagnosis (FD) was different from the initial clinical diagnosis (D1) or the combined diagnosis (CD1) after additional radiological imaging on the day of first evaluation (Table 3). Eighty-five (17.0\%) patients had a change in management, 20 of which $(4.0 \%)$ were considered major (Table 4$)$. A change in management was regarded as major if the clinical strategy changed to a surgical procedure when the initial strategy was conservative. Seventeen patients underwent operation after re-evaluation for presumed appendicitis (1 negative appendectomy), one for an incarcerated umbilical hernia, another for an incarcerated ventral hernia; one patient underwent laparotomy for intestinal obstruction due to adhesions.

Subgroup analysis was performed for sex, age, additional radiological imaging on the day of evaluation, the diagnosis "nonspecific abdominal pain" (NSAP) after initial evaluation (D1 or CD1) and the resident's degree of certainty for the initial clinical diagnosis (Table 5). Female patients had more strategy changes than male patients $(P<0.05)$, and patients older than 16 years had significantly more strategy changes than children $(P<0.0001)$. When patients underwent additional imaging (US and or CT) on the day of initial evaluation, they had fewer diagnostic changes when compared to those who did not $(P=0.0002)$. This, however, did not lead to a significant change in management $(P=0.106)$. Those patients whose abdominal complaints could not be differentiated and were given the diagnosis "nonspecific abdominal pain (NSAP) after initial evaluation $(n=90)$, had more diagnostic changes at re-evaluation $(P=0.006)$. This occurred irrespective of whether they underwent additional radiological imaging on the day of initial evaluation: 39 of the 90 patients $(43.3 \%)$ underwent additional radiological imaging, and 18 of those 39 patients $(47.4 \%)$ had diagnostic changes at re-evaluation. When a resident had a high degree of certainty about his/her initial clinical diagnosis (4 or 5 ), there were significantly fewer diagnostic changes at re-evaluation $(P<0.0001)$. There were no significant differences for major changes in management in any of the subgroups.

Of the 500 patients, only $6(1.2 \%)$ had diagnoses that should preferably have been made on the initial day of evaluation, leading to immediate treatment. Three of those patients had acute perforated appendicitis, one patient had 
Table 1 Diagnosis and management data for 170 excluded patients

\begin{tabular}{|c|c|c|c|c|c|c|c|}
\hline \multirow{3}{*}{ Lost to follow-up } & \multicolumn{3}{|c|}{ No form } & \multicolumn{3}{|c|}{ No show } & \multirow{3}{*}{$\begin{array}{l}\text { Total } \\
23\end{array}$} \\
\hline & \multicolumn{3}{|l|}{15} & \multicolumn{3}{|l|}{8} & \\
\hline & $\mathrm{H}$ & $\mathrm{O}$ & $\mathrm{N}$ & $\mathrm{H}$ & $\mathrm{O}$ & $\mathrm{N}$ & \\
\hline Self-limiting nonspecific abdominal pain & 1 & 1 & 44 & & & 27 & 73 \\
\hline Viral intestinal infection, unspecified & & & 8 & 1 & & 2 & 11 \\
\hline Abdominal pain due to metastasized cancer & 6 & 1 & & 1 & & & 8 \\
\hline Calculus of gallbladder without cholecystitis & $6(5)^{\mathrm{a}}$ & $1(1)^{\mathrm{a}}$ & & & & & 7 \\
\hline Constipation & & & 4 & 1 & & 2 & 7 \\
\hline Acute appendicitis, not perforated & $6(6)^{b}$ & & & & & & 6 \\
\hline Gynecological pathology, NOS & 1 & & 2 & $1(1)^{\mathrm{a}}$ & 1 & & 5 \\
\hline Calculus of kidney and ureter & 3 & & 1 & & & & 4 \\
\hline Irritable bowel syndrome & 1 & & 1 & & 1 & 1 & 4 \\
\hline Pneumonia & 2 & & & & & & 2 \\
\hline Pancreatitis & 2 & & & & & & 2 \\
\hline Extra-uterine gravidity & $2(2)^{\mathrm{b}}$ & & & & & & 2 \\
\hline Gastritis and duodenitis & & 1 & 1 & & & & 2 \\
\hline Cystitis & & & & & & 2 & 2 \\
\hline Peptic ulcer, site unspecified & 1 & & & & & & 1 \\
\hline Acute appendicitis, perforated & $1(1)^{b}$ & & & & & & 1 \\
\hline Inguinal hernia with obstruction, without gangrene & $1(1)^{b}$ & & & & & & 1 \\
\hline Ventral hernia with obstruction, without gangrene & $1(1)^{\mathrm{b}}$ & & & & & & 1 \\
\hline Crohn's disease & 1 & & & & & & 1 \\
\hline Ileus, unspecified & 1 & & & & & & 1 \\
\hline Diverticular disease of the large intestine & $1(1)^{\mathrm{a}}$ & & & & & & 1 \\
\hline Acute tubulo-interstitial nephritis & 1 & & & & & & 1 \\
\hline Functional diarrhea & 1 & & & & & & 1 \\
\hline Benign neoplasm of the colon & 1 & & & & & & 1 \\
\hline Inguinal hernia & & & 1 & & & & 1 \\
\hline Moderate pre-eclampsia & & & & 1 & & & 1 \\
\hline Total & $40(17)$ & $4(1)$ & 62 & $5(1)$ & 2 & 34 & 170 \\
\hline
\end{tabular}

${ }^{a}$ Elective operation

b Acute operation

No form study form was incomplete or not returned; No show patient did not show up for the re-evaluation appointment at the surgical outpatient clinic; $H$ patient was treated at our hospital; $O$ patient was treated at another hospital; $N$ patient did not contact another caregiver for abdominal complaints. Numbers in parentheses indicate the number of patients that underwent operation

an incarcerated ventral hernia without gangrene, one patient had an incarcerated umbilical hernia without gangrene, and another patient had intestinal adhesions with obstruction, but also without gangrene. After recovering from their operations, these patients were discharged from the hospital without complications.

\section{Discussion}

Patients presenting with acute abdominal pain at the ED need to be diagnosed correctly and treated accordingly. As the majority of patients will have self-limiting pathology not requiring surgical intervention, most will not require an admission to the hospital. Patients with abdominal pathology requiring surgical treatment, however, should not be missed. To diagnose patients with abdominal pain, many diagnostic modalities can be used to complement the "basic" clinical evaluation of patient history, physical examination, and blood and urine analysis. Diagnostic measures such as US, CT, laparoscopy, and clinical observation have all been reported in the literature. Ultrasound, for example, can assist in the diagnosis of many gastrointestinal causes of acute abdominal pain [6], and its 
Fig. 2 Summary of the inclusion process for eligible patients

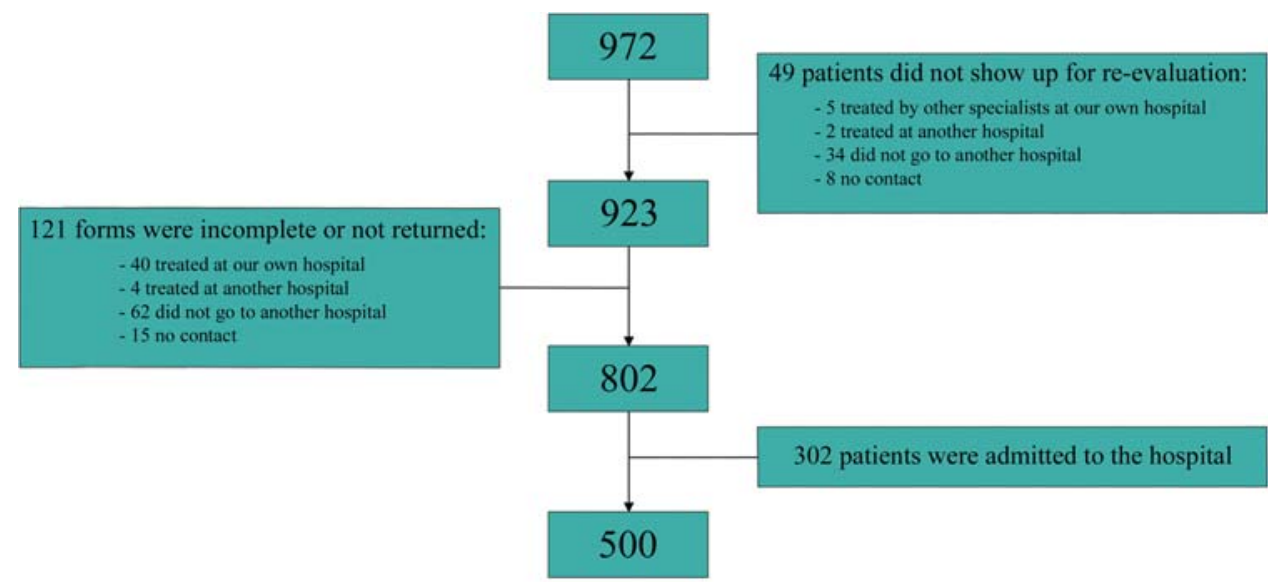

Table 2 The final diagnoses for 500 patients who were not admitted to the hospital but who underwent standard outpatient re-evaluation after presentation at the emergency department for acute abdominal pain

\begin{tabular}{|c|c|c|c|c|c|c|}
\hline \multirow[t]{3}{*}{ Final diagnosis (FD) } & \multicolumn{6}{|c|}{ Patient age } \\
\hline & \multicolumn{2}{|c|}{$>16$ years } & \multicolumn{2}{|c|}{$\leq 16$ years } & \multicolumn{2}{|c|}{ Total } \\
\hline & $n$ & $\%$ & $n$ & $\%$ & $n$ & $\%$ \\
\hline Other and unspecified abdominal pain & 53 & 18.0 & 36 & 17.6 & 89 & 17.8 \\
\hline Constipation & 35 & 11.9 & 46 & 22.4 & 81 & 16.2 \\
\hline Viral intestinal infection, unspecified & 25 & 8.5 & 45 & 22.0 & 70 & 14.0 \\
\hline Nonspecific mesenteric lymphadenitis & 3 & 1.0 & 33 & 16.1 & 36 & 7.2 \\
\hline Cystitis & 25 & 8.5 & 8 & 3.9 & 33 & 6.6 \\
\hline Calculus of kidney and ureter & 27 & 9.2 & 0 & 0 & 27 & 5.4 \\
\hline Calculus of gallbladder without cholecystitis & 21 & 7.1 & 0 & 0 & 21 & 4.2 \\
\hline Diverticular disease of intestine & 20 & 6.8 & 0 & 0 & 20 & 4.0 \\
\hline Gynecological pathology, NOS & 17 & 5.8 & 1 & 0.5 & 18 & 3.6 \\
\hline Gastritis and duodenitis & 12 & 4.1 & 4 & 2.0 & 16 & 3.2 \\
\hline Acute appendicitis, not perforated & 8 & 2.7 & 6 & 2.9 & 14 & 2.8 \\
\hline Ileocaecitis & 6 & 2.0 & 6 & 2.9 & 12 & 2.4 \\
\hline Pneumonia, organism unspecified & 1 & 0.3 & 7 & 3.4 & 8 & 1.6 \\
\hline Acute tubulo-interstitial nephritis & 5 & 1.7 & 1 & 0.5 & 6 & 1.2 \\
\hline Myalgia (abdominal wall) & 3 & 1.0 & 2 & 1.0 & 5 & 1.0 \\
\hline Bronchitis, not specified as acute or chronic & 0 & 0 & 4 & 2.0 & 4 & 0.8 \\
\hline Peptic ulcer, site unspecified & 3 & 1.0 & 0 & 0 & 3 & 0.6 \\
\hline Other & 31 & 10.5 & 6 & 2.9 & 37 & 7.4 \\
\hline Total & 295 & & 205 & & 500 & \\
\hline
\end{tabular}

NOS not otherwise specified

routine use by surgeons for such patients has been shown to increase diagnostic accuracy [7]. Computer tomography has also been reported to increase diagnostic accuracy and reduce hospital admissions by $24 \%$ [8], and it is said to be the best predictor of the need for an urgent intervention [9]. The use of cross-sectional imaging for all patients presenting with acute abdominal pain, however, is costly, time consuming, and, in the case of $\mathrm{CT}$, exposes patients to ionizing radiation. The last issue is important as the growing use of $\mathrm{CT}$ is steadily increasing the collective dose of medical radiation to which patient populations are subjected. Even though the cancer risk from an abdominal CT is small for the individual, the increasing use of the modality may create a future health concern, especially for children [10]. The indications for ordering a CT should therefore always be carefully scrutinized by medical personnel, especially when other diagnostic modalities can attain similar results [11].

Laparoscopy can also accurately distinguish patients that require surgery from those that can be treated 
Table 3 Changes in the diagnosis after standard outpatient re-evaluation

\begin{tabular}{|c|c|c|c|c|c|c|c|c|c|}
\hline \multirow[b]{2}{*}{$\mathrm{D} 1$ or $\mathrm{CD} 1$} & \multicolumn{9}{|c|}{ Final Diagnosis (FD) } \\
\hline & VII & NML & GD & AA & $\mathrm{CO}$ & NSAP & GYN & Other & Total \\
\hline Viral intestinal infection, unspecified (VII) & & 2 & 2 & 2 & 5 & 6 & 1 & 10 & 28 \\
\hline Nonspecific mesenteric lymphadenitis (NML) & 1 & & & 2 & 1 & 2 & & 1 & 7 \\
\hline Acute appendicitis, not perforated (AA) & 5 & 4 & 1 & & 2 & 2 & & & 14 \\
\hline Constipation (CO) & & & & 1 & & 8 & 3 & 5 & 17 \\
\hline Calculus of kidney and ureter & & & & & & 2 & & 5 & 7 \\
\hline Nonspecific abdominal pain (NSAP) & 6 & 1 & 2 & 2 & 10 & & 2 & 11 & 34 \\
\hline Other & 2 & 1 & 2 & 1 & 2 & 17 & 1 & 15 & 41 \\
\hline Total & 14 & 8 & 7 & 8 & 20 & 37 & 7 & 47 & 148 \\
\hline
\end{tabular}

$D 1$ first clinical diagnosis; $C D 1$ "combined" diagnosis after additional radiological imaging (on the day of initial evaluation); $G D$ gastritis and duodenitis; GYN gynecological pathology; NOS not otherwise specified

Table 4 Strategy changes after standard outpatient re-evaluation

\begin{tabular}{|c|c|c|c|c|c|}
\hline \multirow[b]{2}{*}{$\mathrm{S} 1$ or $\mathrm{CS} 1$} & \multicolumn{5}{|c|}{ Final strategy after re-evaluation } \\
\hline & LAP & $\mathrm{ADM}$ & OPFU & COS & Total \\
\hline Laparotomy (LAP) & & & 2 & & 2 \\
\hline Outpatient re-evaluation & 20 & 5 & & 52 & 77 \\
\hline Consultation of other specialty (COS) & & & 5 & 1 & 6 \\
\hline Total & 20 & 5 & 7 & 53 & 85 \\
\hline
\end{tabular}

S1 first clinical strategy; $C S 1$ "combined" strategy after additional radiological imaging (on the day of initial evaluation); $A D M$ admission; $O P F U$ outpatient follow-up

Table 5 Subgroup analysis for diagnostic and strategy changes

\begin{tabular}{llllllll}
\hline & $\mathrm{N}$ & $\Delta \mathrm{D}$ & $\% \Delta \mathrm{D}$ & $\Delta \mathrm{S}$ & $\% \Delta \mathrm{S}$ & $\mathrm{M} \Delta \mathrm{S}$ & $\% \mathrm{M} \Delta \mathrm{S}$ \\
\hline All patients & 500 & 148 & 29.6 & 85 & 17.0 & 20 & 4.0 \\
Male & 210 & 54 & 25.7 & $27^{*}$ & 12.9 & 10 & 4.8 \\
Female & 290 & 94 & 32.4 & $58^{*}$ & 20.0 & 10 & 3.4 \\
$>16$ years & 295 & 87 & 29.5 & $67 * *$ & 22.7 & 11 & 3.7 \\
$\leq 16$ years & 205 & 61 & 29.8 & $18^{* *}$ & 8.8 & 9 & 4.4 \\
US/CT on day 0 & 164 & $30^{*}$ & 18.3 & 21 & 12.8 & 5 & 3.0 \\
No US/CT on day 0 & 336 & $118^{*}$ & 35.1 & 64 & 19.0 & 15 & 4.5 \\
NSAP & 90 & $38^{*}$ & 42.2 & 13 & 14.4 & 2 & 2.2 \\
Non NSAP & 410 & $110^{*}$ & 26.8 & 72 & 17.6 & 18 & 4.4 \\
Degree of certainty & $231^{\mathrm{a}}$ & $50^{* *}$ & 21.6 & 33 & 14.3 & 8 & 3.5 \\
$\quad$ (4-5) & & & & & & & \\
Degree of certainty & $206^{\mathrm{a}}$ & $81^{* *}$ & 39.3 & 42 & 20.4 & 12 & 5.8 \\
$(1-3)$ & & & & & & & \\
\hline
\end{tabular}

${ }^{\text {a }}$ Missing data: $n=63$

$* P<0.05$

** $P<0.0001$

$\Delta D$ change in diagnosis; $\Delta S$ change in strategy; $M \Delta S$ major change in strategy

conservatively [12], and it has been proposed as routine management for patients with acute abdominal pain for whom the decision to operate is uncertain [13].
Nevertheless, laparoscopy in itself is an operation, and can be regarded as too invasive as a first-line diagnostic measure when similar accuracy can be achieved without an operation.

Hospital admission for patients with equivocal abdominal complaints has been a common practice for many years. The effectiveness of this practice is limited because most patients ultimately have NSAP for which an admission to hospital is in fact not required [14-16]. In recent years the percentage of hospital admissions for patients with acute abdominal pain has decreased, possibly as a result of advances in diagnostic technology and improved ED faculty presence [1].

Another method often used to help distinguish surgical pathology from mild self-limiting disease in patients with equivocal abdominal pain is outpatient re-evaluation. Patients with abdominal pathology requiring surgery who initially present during the early stages of the disease will become more easily identifiable, whereas the symptoms will regress in those patients with self limiting disease allowing them to be safely discharged from follow-up.

Outpatient re-evaluation for patients with equivocal abdominal complaints has not been reported extensively in the literature. Only one study comparing outpatient followup to active clinical observation for patients with NSAP in the ED concluded that outpatient evaluation seems to be a 
safe option that is not accompanied by an increased incidence of complications [17]. The present study is the first to report the value of standard outpatient re-evaluation for patients that are presumed not to require a hospital admission after evaluation at the ED for acute abdominal complaints. After standard re-evaluation $30 \%$ of the patients had a different final diagnosis than the diagnosis initially given after evaluation at the ED. A change in management was seen in $17 \%$ of the cases after re-evaluation, and $4 \%$ of the patients were later admitted to hospital for surgery. These are important changes from the patient's point of view, demonstrating that standard outpatient reevaluation is a valuable method that improves diagnostic accuracy and helps to select the proper management strategies in this patient population. The initial management decisions made by the evaluating physician at the ED regarding whether patients should receive additional imaging or be admitted to the hospital were not scrutinized in this study. The study was designed to mimic daily practice, and allowed for these management decisions to be made just as they are in daily routine where basic clinical judgment plays a fundamental role. Apparently these clinical assessments are precise enough to triage accurately without detrimental effects for the patient. Only 6 patients (1.2\%) had diagnoses that should preferably have been made at initial evaluation. This however did not lead to increased morbidity.

\section{Conclusions}

The present study supports the hypothesis that serial outpatient re-evaluation is safe, and will improve diagnostic accuracy and facilitate proper treatment selection for patients that are not admitted to the hospital after surgical consultation for acute abdominal pain at the emergency department.

Open Access This article is distributed under the terms of the Creative Commons Attribution Noncommercial License which permits any noncommercial use, distribution, and reproduction in any medium, provided the original author(s) and source are credited.

\section{References}

1. Powers RD, Guertler AT (1995) Abdominal pain in the ED: stability and change over 20 years. Am J Emerg Med 13:301-303

2. Hayes R (2004) Abdominal pain: general imaging strategies. Eur Radiol 14(Suppl 4):L123-L137

3. Leschka S, Alkadhi H, Wildermuth S et al (2005) Multi-detector computed tomography of acute abdomen. Eur Radiol 15:2435-2447

4. World Health Organization. International statistical classification of diseases and related health problems: 10th Revision (2007) Available at http://apps.who.int/classifications/apps/icd/icd10online/ Accessed December 82009

5. Veen MR, Lardenoye JW, Kastelein GW et al (1999) Recording and classification of complications in a surgical practice. Eur $\mathrm{J}$ Surg 165:421-424

6. Puylaert JB (2003) Ultrasonography of the acute abdomen: gastrointestinal conditions. Radiol Clin North Am 41:1227-1242

7. Allemann F, Cassina P, Rothlin M et al (1999) Ultrasound scans done by surgeons for patients with acute abdominal pain: a prospective study. Eur J Surg 165:966-970

8. Rosen MP, Sands DZ, Longmaid HE III et al (2000) Impact of abdominal CT on the management of patients presenting to the emergency department with acute abdominal pain. AJR Am J Roentgenol 174:1391-1396

9. Gerhardt RT, Nelson BK, Keenan S et al (2005) Derivation of a clinical guideline for the assessment of nonspecific abdominal pain: the Guideline for Abdominal Pain in the ED Setting (GAPEDS) Phase 1 Study. Am J Emerg Med 23:709-717

10. Brenner DJ, Hall EJ (2007) Computed tomography-an increasing source of radiation exposure. N Engl J Med 357:22772284

11. Hall EJ, Brenner DJ (2008) Cancer risks from diagnostic radiology. Br J Radiol 81:362-378

12. Vander Velpen GC, Shimi SM, Cuschieri A (1994) Diagnostic yield and management benefit of laparoscopy: a prospective audit. Gut 35:1617-1621

13. Paterson-Brown S (1993) Emergency laparoscopic surgery. Br J Surg 80:279-283

14. de Dombal FT (1979) Acute abdominal pain-an O.M.G.E. survey. Scand J Gastroenterol Suppl 5629-5643

15. Laurell H, Hansson LE, Gunnarsson U (2006) Diagnostic pitfalls and accuracy of diagnosis in acute abdominal pain. Scand $\mathrm{J}$ Gastroenterol 41:1126-1131

16. Sheridan WG, White AT, Havard T et al (1992) Non-specific abdominal pain: the resource implications. Ann R Coll Surg Engl 74:181-185

17. Onur OE, Guneysel O, Unluer EE et al (2008) "Outpatient follow-up" or "active clinical observation" in patients with nonspecific abdominal pain in the emergency department. A randomized clinical trial. Minerva Chir 63:9-15 